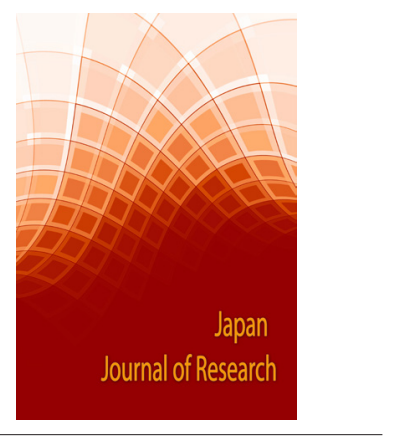

Correspondence

Georg Voelcker

Institute of Biochemistry II, Goethe University Frankfurt Medical School, 60590 Frankfurt, Germany.

E-mail: voelcker@biochem2.de

Tel: +496963015652

Fax: +496963015577

\footnotetext{
- Received Date: 25 Mar 2020;

- Accepted Date: 18 Apr 2020;

- Publication Date: 23 Apr 2020.
}

Keywords

Cyclophosphamide, Drugs, Oxazaphosphorine Cytostatics, Tumors, Therapeutic effect, $\mathrm{OHCP}$.

Copyright

(C) 2020 Science Excel. This is an openaccess article distributed under the terms of the Creative Commons Attribution 4.0 International license.

\title{
The Real Mechanism of Action of Cyclophosphamide and Other Oxazaphosphorine Cytostatics
}

\author{
Georg Voelcker \\ Institute of Biochemistry II, Goethe University Frankfurt Medical School, 60590 Frankfurt, Germany
}

Cyclophosphamide (CP), an alkylating cytostatic that has been successfully applied in the clinic for more than 50 years, is a prodrug that is hydroxylated in the liver by $\mathrm{P} 450$ enzymes to 4-hydroxy-cyclophosphamide (OHCP). OHCP forms an equilibrium mixture with its tautomeric aldehyde aldophosphamide (ALDO). Like CP itself, OHCP and ALDO are prodrugs for the actual alkylating phosphoreamidemustard (PAM), which stops cell proliferation by DNA alkylation.

The therapeutic index, that is the ratio from the amount of a drug that causes toxicity to the amount that causes the therapeutic effect is a measure of the therapeutic efficiency of a drug. In this special case the ratio of the dose which kills $50 \%$ of the rats (LD50) to the dose which cures $50 \%$ of Yoshida ascites sarcoma-bearing rats (CD50) was determined for $\mathrm{CP}, \mathrm{OHCP}$ and PAM. The therapeutic index (LD50/CD50) was determined to be 120 for $\mathrm{CP}$ and OHCP but only 3.5 for PAM [1]. Why is the therapeutic efficiency of the prodrugs $\mathrm{CP}$ and $\mathrm{OHCP}$ more than 30 times greater than that of PAM which is the metabolite, for the actually therapeutic alkylation reaction?

Limb perfusion experiments in rats were carried out. Rats bearing solid growing Yoshida ascites tumor cells transplanted into the $\mathrm{m}$. gastrocnemius were perfused for 30 minutes via a. epigastrica and v. epigastrica with an oxygenated hemoglobin solution containing $\mathrm{OHCP}$ the concentration of which was $1 \mu \mathrm{M}$. During perfusion, the blood supply to the leg was disconnected from the body circulation by a tourniquet. No rat was cured by this method. Perfusion with higher OHCP concentrations was not possible due to the local toxicity of OHCP. But contrary to therapy by limb perfusion 5 of 10 rats with the tumor in the same place were cured with a single dose of $125 \mathrm{mg} / \mathrm{kg}$ $\mathrm{CP}$ administered intraperitoneally [2]. The highest concentration of OHCP in blood samples drawn from the a. epigastrica after $\mathrm{CP}$ administration was $0.05 \mu \mathrm{mol} / \mathrm{ml}$ and the area under curve (auc) calculated from the measurements was $5.4 \mu \mathrm{mol}$ min compared to a maximum OHCP concentration of $1 \mu \mathrm{mol} / \mathrm{ml}$ and an auc of 30 $\mu \mathrm{mol}$ min in the perfusion experiment. Why is the therapeutic success due to regional perfusion worse - better not measurable - despite a 20 -fold higher OHCP concentration and 5.6-fold higher auc than after CP administration?

These results demonstrate that OHCP in these experiments is only the carrier of cancer selectivity if it reaches the tumor transplanted into the $\mathrm{m}$. gastrocnemius after passage through the body.

\section{Why is body passage so important?}

The previous ideas about the mechanism of action of $\mathrm{CP}$ which are based on the results of cell culture experiments and which are out of date because they are not adapted to the new knowledge of biochemistry, assume that PAM is formed by spontaneous $B$-elimination of acrolein from ALDO [3] but this is only true for in vitro experiments [4].

By the outdated notion of the mechanism of action of $\mathrm{CP}$ the cancer selectivity is due to enrichment of PAM in tumor cells due to lower activity of ALDOdetoxifying aldehyde dehydrogenases $(\mathrm{ADH})$ in tumor cells than in normal cells [5]. ADH oxidize ALDO to the non-cytotoxic carboxyphosphamide.

Two events speak against the assumption of an accumulation of PAM in tumor cells through selective stronger detoxification in normal cells as the cause of the cancer selectivity of CP.

1. Failure of the drug Glufosfamide. In the case of Glufosfamide, the alkylating metabolite of Ifosfamide that is IPAM is coupled to glucose. Due to the tumor cell's increased need for glucose, IPAM is enriched in tumor cells. The mechanism of action of Ifosfamide is the same as that of CP.

2. The fact that after $\mathrm{CP}$ injection in humans or animals, PAM is not produced by spontaneous $ß$-elimination of acrolein but by enzymatic cleavage of ALDO. Cleaving enzymes are phosphodiesterases (PDE) that cleave ALDO in PAM and 3-hydroxypropanal (HPA) [4]. Acrolein is not formed in the metabolism of $\mathrm{CP}$ when administered in human or animals but HPA.

The latter explains the therapeutic failure of the regional perfusion of the tumor-bearing limb. The perfusion solution containing OHCP does not contain the PDE necessary for the formation of PAM. The amount of PAM that is formed during 
the 30 min perfusion by spontaneous $\beta$-elimination of acrolein from ALDO is too small for an effective therapy. This does not only apply to therapy by regional perfusion, but would also apply to any therapy with CP, because the half-life for the spontaneous formation of PAM by $ß$ elimination of acrolein in protein-free rat serum ultrafiltrate is 23 hours, but in serum containing PDE only 20 minutes [4]. This shows that the rapid enzyme catalyzed cleavage of ALDO with the formation of PAM and HPA is a prerequisite for the cytotoxic effects of CP and other oxazaphosphorine cytostatics like Ifosfamide (IF).

The enzymatic cleavage of ALDO to form PAM and HPA is important for another reason that makes CP exceptional as an alkylating cytostatic. HPA - which becomes acrolein by elimination of water - is a pro-apoptotic aldehyde that promotes apoptosis by inhibiting the antiapoptotic proteins Bcl-xL and Bcl-2, by inhibiting NFKB activation and by activating mitogen-activated protein kinase (MAPK) [6]. In view of the fact that the cell-toxic event during therapy with $\mathrm{CP}$ is not DNA damage due to alkylation, but rather apoptosis triggered by DNA damage [7], the sheme figure.1 explained in the legend describes the mechanism of action of $\mathrm{CP}$ and other oxazaphophorine cytostatics.

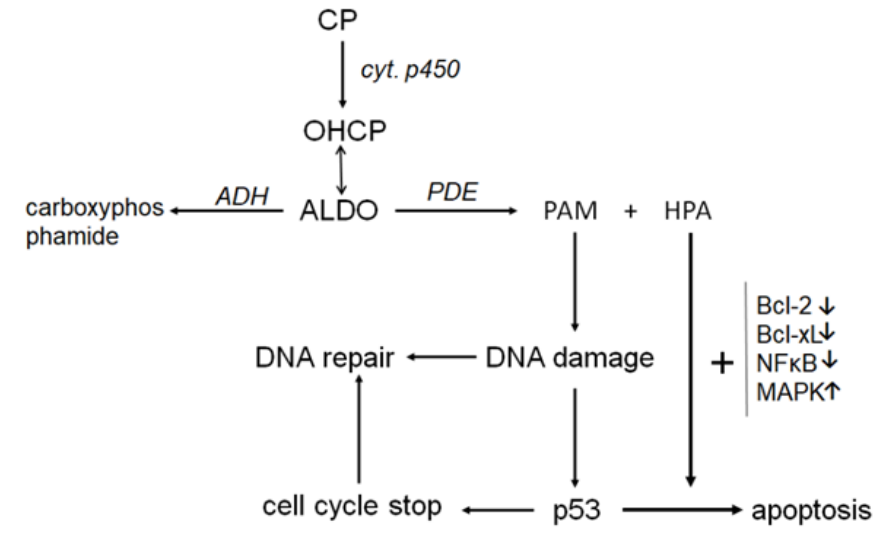

Figure 1.

Cyclophosphamide is hydroxylated by $\mathrm{P} 450$ enzymes in the liver to the corresponding hydroxyl derivative (OHCP) which is in equilibrium with aldophosphamide (ALDO), ALDO is the pharmacologic active metabolites. A part of ALDO is oxidized by aldehyde dehydrogenase $(\mathrm{ADH})$ to the non-toxic carboxyphosphamide. The amount of ALDO not oxidized, is decomposed by phosphodiesterases (PDE) to the alkylating phosporeamidemustard (PAM) and 3-hydroypropanal (HPA). PAM damages DNA by alkylation. The alkylated DNA is either repaired immediately or - if this is not possible - the tumor suppressor protein p53 is activated which induces cell cycle stop to give the cell time to repair the damage. If DNA repair is not possible p53 induces apoptosis, which is - and this is special for CP and other oxazaphosphorines - enhanced by HPA which supports apoptosis by inhibiting the anti-apoptotic proteins $\mathrm{Bcl}-2$ and $\mathrm{Bcl}-\mathrm{xL}$, and by inhibiting NFKB activation. Additional, HPA promotes apoptosis by enhancing mitogen activated protein kinase (MAPK) activities by enhancing JNK and p38 phosphorylation.
The validity of the proposed mechanism of action was checked in chemotherapy experiments in CD2F1 mice bearing advanced subcutaneously growing P388 mice leukemia cells. The therapy experiments were carried out with the IF derivatives aldo-ifosfamideperhydrothiazine (IAP) and aldo-mesyl-ifosfamide-perhydrothiazine (SUM-IAP) [8]. According to the proposed mechanism of action, cells are only eradicated when DNA damage produced by the alkylating agent is not repaired by cellular repair mechanisms. IAP and SUMIAP spontaneously hydrolyze to the corresponding aldo-ifosfamide bypassing the toxic 4-hydroxyifosfamide. IAP contains the alkylating function of IF, namely two 2-chloroethyl groups which form DNA interstrand crosslinks that are repaired very efficiently whereas in In the SUM-IAP molecule one chlorine is substituted by a mesyl-ethyl group which forms intrastrand cross links that are poorly repairable (http://www.atdbio.com/content/16/Nucleic-acid-drug-interactions). A low dose $(0.29 \mathrm{mmol} / \mathrm{kg}$ corresponding to $115 \mathrm{mg} / \mathrm{kg}$ IAP) of IAP and SUM-IAP was administered 5 times on days 7-11 after tumor transplantation. IAP shows only marginal antitumor activity measured as growth delay of only 2 days compared to a non-treated control group, whereas after treatment with SUM-IAP tumor burden was decreased below detection level for five days [8].

\section{References}

1. Brock N. Comparative pharmacologic study in vitro and in vivo with cyclophosphamide (NSC-26271), cyclophosphamide metabolites, and plain nitrogen mustard compounds. Cancer Treat Rep. 1976; 60(4): 301308.

2. Skupin W. Die regionale Perfusion des isolierten tumortragenden Rattenbeins mit stabilisierten aktivierten Cyclo-Phosphamiden in Kombination mit Protektorthiolen. Inaugural-Dissertation zur Erlangung des Doktorgrades beim Fachbereich Veterenärmedizin der Justus-Liebig-Universität Giesen 1990.

3. Low JE, Borch RF, Sladek NE. Further studies on the conversion of 4-hydroxyoxazaphosphorines to reactive mustards and acrolein in inorganic buffers.Cancer Res. 1983;43(12 Pt 1):5815-5820.

4. Voelcker G. Enzyme catalyzed decomposition of 4-hydroxycyclophosphamide, The Open conference Proceeding Journal. 2017;8:44-51.

5. Sládek NE, Kollander R, Sreerama L, et al. Cellular levels of aldehyde dehydrogenases (ALDH1A1 and ALDH3A1) as predictors of therapeutic responses to cyclophosphamide-based chemotherapy of breast cancer: a retrospective study. Rational individualization of oxazaphosphorine-based cancer chemotherapeutic regimens. Cancer Chemother Pharmacol. 2002;49(4):309-321.

6. Iyer C, Kosters A, Sethi G, et al. Probiotic Lactobacillus reuteri promotes TNF-induced apoptosis in human myeloid leukemia-derived cells by modulation of NF-kappa B and MAPK signalling. Cell Microbiol. 2008;10(7):1442-1452.

7. Schwartz PS, Waxman DJ. Cyclophosphamide induces caspase 9-dependent apoptosis in $9 \mathrm{~L}$ tumor cells. Mol Pharmacol. 2001;60(6):1268-1279.

8. Voelcker G. Influence of the alkylating function of aldo-Ifosfamide on the anti-tumor activity. Anticancer Drugs. 2018;29(1):75-79. 